\title{
A Modified Learning Algorithm for Backpropagation Network
}

\author{
Yao Zhang, Grant E. Hearn and Pratyush Sen \\ Dept. of Marine Technology, The University of Newcastle upon Tyne \\ Newcastle upon Tyne NE1 7RU, U.K.
}

\begin{abstract}
A modified backpropagation (MBP) algorithm is proposed in this paper. The MBP algorithm is based on the idea that both the error $E_{p}$ and its change $\Delta E_{p}$ can be applied as information to update weights. It can be seen from Fig. 1 that when $E_{p}$ curve is convex, the signs of $E_{p}$ and $\Delta E_{p}$ slopes are the same. When $E_{p}$ curve becomes concave, the signs are opposite. Therefore, if $\Delta E_{p}$ slope is added to or subtracted from $E_{p}$ slope as appropriate, the network training is expected to speed up. This idea can be expressed as

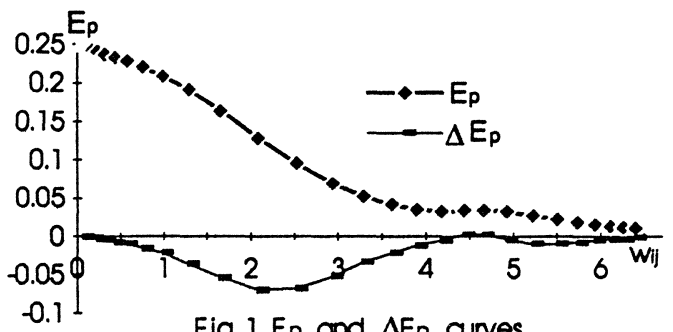

$$
\Delta w_{i j}=-\eta \frac{\partial E_{p}}{\partial w_{i j}}+\operatorname{sign}\left(\frac{\partial \Delta E_{p}}{\partial w_{i j}}\right) \cdot \xi \cdot \frac{\partial \Delta E_{p}}{\partial w_{i j}}
$$

where $\xi$ is a constant defined as an accelerator. For clarity, we define $\mathbf{i}, \mathbf{j}$ and $\mathbf{k}$ as the unit in output, hidden and input layers. The derivatives of $\Delta E_{p}$ with respect to $w_{i j}$ and $w_{j k}$ are given as

$$
\begin{aligned}
& \frac{\partial \Delta E_{p}}{\partial w_{i j}}=\left[\frac{\partial^{2} o_{p i}}{\partial w_{i j}^{2}} \cdot \frac{\partial E_{p}}{\partial o_{p i}}+\left(\frac{\partial o_{p i}}{\partial w_{i j}}\right)^{2}\right] \Delta w_{i j} \\
& \frac{\partial \Delta E_{p}}{\partial w_{j k}}=\sum_{i}\left[\left(\frac{\partial o_{p i}}{\partial w_{i j}} \cdot \frac{\partial o_{p i}}{\partial w_{j k}}+\frac{\partial E_{p}}{\partial o_{p i}} \cdot \frac{\partial^{2} o_{p i}}{\partial w_{i j} \partial w_{j k}}\right) \Delta w_{i j}\right]
\end{aligned}
$$

Simulations are carried out on the XOR, parity, encoding and symmetry problems. Results show that when compared to the conventional backpropagation (CBP) algorithm, MBP can reduce the training iterations by from $25 \%$ to $90 \%$, depending on different problems.

Another attractive feature of MBP algorithm is that it is convergent when starting with equal or even zero initial weights. This eliminates the guesswork of finding proper initial weights for the network to avoid local minimum. Table 1 gives some of the MBP simulation results starting with zero initial weights. The CBP iterations listed in the table are the average of several training sessions with different random initial weights ( $\eta$ is the leaming rate and $\alpha$ is momentum factor).

Table 1. MBP training with zero initial weights (compared to CBP training)

\begin{tabular}{|c|c|c|c|c|c|}
\hline$\eta$ & $\alpha$ & \multicolumn{2}{|c|}{ Training iterations in XOR problem } & \multicolumn{2}{c|}{ Training iterations in Symmetry problem } \\
\cline { 3 - 6 } & & $\operatorname{MBP}(\xi=10)$ & CBP (average) & MBP $(\xi=4.0)$ & CBP (average) \\
\hline 0.1 & 0.1 & 17380 & 57860 & 411120 & 532160 \\
\hline 0.9 & 0.9 & 220 & 520 & 6080 & 8640 \\
\hline
\end{tabular}

